Keywords: Twin boundaries, Nye tensor, dislocation density

\title{
Quantitative assessment of the driving force for twin formation utilizing Nye tensor dislocation density mapping
}

\author{
A.C. Leff ${ }^{1}$ and M.L. Taheri ${ }^{1 *}$ \\ ${ }^{1}$ Department of Materials Science \& Engineering, Drexel University, Philadelphia, PA \\ *Author to whom correspondence should be addressed: mtaheri@ coe.drexel.edu
}

\section{Abstract}

8 Transmission electron microscopy was coupled with precession electron diffraction methods to 9 determine the role of local defect distributions in the formation and growth behavior of twin 10 nuclei. Three distinct cases were observed that are indicative of three separate formation 11 mechanisms, two as the result of annealing and the third as a result of deformation. While all of 12 the observed cases are consistent with the proposed mechanisms for twin formation found in 13 literature, this study marks the first experimental evidence to support all three mechanisms 14 occurring simultaneously in the same microstructure.

Through thermomechanical processing, the microstructure of metals can be tailored to the specific requirements of a given application; properties such as toughness, corrosion resistance, and even radiation tolerance can be enhanced by altering grain boundary density and connectivity [1-4]. Increasing the fraction of coherent twin and other low energy grain boundary configurations is known to improve overall material performance. Empirical methods of grain boundary engineering (GBE) have been developed to enhance the fraction of twin and twinrelated boundaries [1,5-7]. Understanding twin nucleation and propagation with respect to experimental parameters, such as local strain gradients, is critical for improving the design of processing methods to enhance the properties of structural materials, as the mechanisms by which twins nucleate and propagate during thermomechanical processing are still not well understood.

The leading models for annealing twin formation are those put forth by Gleiter [8], Myers and Murr [9], and Mahajan et al. [10]. Both Gleiter and Mahajan et al. 's models are rooted in the concept that growth accidents at the $\{111\}$ planes of migrating grain boundaries result in atoms landing in twinned sites relative to the orientation of the growing grain, creating coherent twin nuclei that trail behind the migrating grain boundary. Both developed quantitative models for calculating the twin density based on the probability of growth accidents occurring as a function of annealing temperature, the melting temperature of the material and its intrinsic stacking fault energy of a given FCC metal. Cahoon et al. [11] introduced modifications to both models to more accurately predict the annealing twin density as a function of cold work, moving one step 
closer to a predictive understanding of microstructural development during industrial thermomechanical processing. A different model, proposed by Myers and Murr [9], postulates that twin domains form by a popping out mechanism driven by the formation of Shockley partial dislocations at high angle grain boundaries, which facilitate the dissociation of the existing grain boundary into two twins, one coherent and one incoherent. These twins "pop out" from the original boundary, leaving a $\Sigma 9$ in its place. This is just one possible structure for such a boundary dissociation mechanism. The key distinction that separates this model from the growth accident model is that it does not require boundary migration in order for the twin nuclei to form. All viable models of deformation twinning agree that twin nuclei form through the interaction of Shockley partial dislocations with $\{111\}$ planes $[12,13]$.

\section{6}

Although a myriad of studies have analyzed the conditions that promote twin formation [14-18] and highly twinned GBE microstructures [1,2,19-21], the exact conditions that promote twin nucleation are still unclear, however, especially at the nano-scale. While it is clear that dislocations play a role in twin nucleation, both in terms of forming the twin structure itself via the migration of partial dislocations and as a source of energy driving the process [14-16], defect gradients and misorientation relationships that best promote twin nucleation are still unknown. Recent studies performed on $\mathrm{Cu}$ and Ni-based materials have found that twins nucleate preferentially at fast-moving grain boundaries [15-17], consistent with the growth accident model. Additionally, the twin orientation was found to be determined by which <111> axis is closest to the growth direction, which further supports the growth accident model [15]. Jin, et al. [16] observed twin formation on a grain by grain basis using in situ annealing in an SEM equipped with EBSD and found that twins only form at boundaries migrating into strained regions and that there was a strong correlation between individual boundary migration rates and twin density. These studies indicate that twinning occurs most frequently at the points of highest strain and that therefore, maximizing microstructural deformation and using a high temperature anneal performed with a fast heating rate ought to promote a GBE microstructure.

Other studies performed on $\mathrm{Cu}, \mathrm{Ni}$ alloys, and stainless steel, however, have reported the opposite observation: that twinning occurs in areas of low strain [14,20,21] and at low annealing temperatures $[14,18]$ and heating rates [18]. They suggest that twinning is promoted under low strain/low temperature conditions due to a more energetically favorable orientation for boundary migration that allows recrystallization to continue. This is similar to the popping out model for twin nucleation [9], in which twins form at high energy boundaries in order to reduce the internal energy of the system. It is also consistent with the observation of secondary recrystallization resulting in high fractions of CSLs [17] and twin-related domains [22] under certain conditions. This finding is also in agreement with the observation that the low strain/low temperature annealing twin formation conditions coincide with the regime in which strain induced boundary migration is the dominant recrystallization mechanism. As such, twinning occurs at the interfaces between the recrystallized and strained regions in order to provide a more energetically favorable orientation for migration. Such a mechanism for the propagation of CSL boundaries was first suggested by V. Randle [19], who concluded that the interaction of the twin domains produces the higher order CSL boundaries present in GBE microstructures. 
Based on these contrasting results, two regimes of localized plastic strain that promote twin formation prevail: a high boundary migration driving force regime, in which the growth accidents mechanism is dominant, and a low driving force regime, in which a boundary decomposition phenomenon, such as that which the popping out describes, is dominant. To test this hypothesis, precession electron diffraction (PED) was used in conjunction with Nye tensor analysis to calculate and map dislocation densities in order to determine localized microstructural conditions required for twin nucleation characterize twin nucleation sites in $\mathrm{Cu}$. While various processing approaches can be used to increase the fraction of twin boundaries, a method based on the low driving force regime is more promising for the production of GBE microstructures with a highly inter-connected $\Sigma 3^{\mathrm{n}}$ grain boundary network [20-22]. The results presented in this paper connect twin formation phenomena to local dislocation-based driving force, and thus clarify which of the mechanisms play a role in the formation of twin- related microstructure networks.

90 Two distinct thermomechanical processing treatments were applied to >99.99\% pure $\mathrm{Cu}$ (McMaster Carr ${ }^{\mathrm{TM}}$ ) to produce partially recrystallized microstructures. The first treatment consisted of a $50 \%$ cold rolling reduction followed by a 10 minute anneal at $400^{\circ} \mathrm{C}$. The second, iterative treatment consisted of three iterations of cold rolling reduction to $25 \%$ followed by 10 minute anneals at $400^{\circ} \mathrm{C}$. TEM specimens were prepared by traditional grinding and polishing methods followed by electropolishing using a Struers ${ }^{\mathrm{TM}}$ TenuPOL5 jet polisher. A JEOL ${ }^{\mathrm{TM}} 2100$ $\mathrm{LaB}_{6}$ TEM equipped with NanoMEGAS ASTAR ${ }^{\mathrm{TM}}$ orientation mapping system was used for characterization of the regions around newly formed twin boundaries. All scans were performed using a step size of $10.4 \mathrm{~nm}$. Local dislocation density values and grain-average dislocation density values were estimated and mapped using a Nye tensor algorithm [23]. Jiang, et al. discuss the effectiveness of the Nye tensor for mapping dislocation densities in their recent paper [24]. Although the Nye tensor only accounts for geometrically necessary dislocations (GNDs), it has been shown that as the length scale on which the analysis is performed is reduced, the fraction of dislocations considered to be geometrically necessary, rather than statistically stored, increases logarithmically [23,25,26]. As a result, in addition to the dislocations traditionally considered to be GNDs, a large fraction of the statistically stored dislocations ought to be accessible by this method at the nanometer length-scale. This provides a new, more accurate way beyond the means of EBSD [27] to measure stored energy as a driving force for recrystallization. A nearest neighbor radius of two was used for the analysis in order to reduce noise due to angular resolution limits - approximately $0.3^{\circ}$ [28]. An error simulation was run in order to estimate the level of uncertainty in a given density value based on these input parameters. It was found that the median error for a given value was $6.81 \times 10^{14} \mathrm{~m}^{-2}$. For a complete description of this simulation see the supplemental material. Additionally a low-pass discrete Fourier filter was applied to remove some of the high frequency noise due to uncertainty introduced by this angular resolution limit. Stored energy density $(G)$ was calculated from dislocation density $(\rho)$ according to the following equation:

$$
G=\rho \mu b^{2} / 2
$$

where $\mu$ is the shear modulus and $b$ is the magnitude of the Burgers vector, in order to better quantify the driving forces for boundary migration and twin nucleation. 
Three distinct local strain conditions, or dislocation density gradients, were observed in the regions surrounding new twins, indicative of formation by growth accidents, popping out, and deformation twinning, respectively. In the first case (Figure 1) twins grew into the low dislocation density grain at an interface between two grains with a gradient in densities across them. This observation is in agreement with the growth accident model of twin formation; it appears that the interface between grains 1 and 2 (Fig 1a) migrated rightward with atoms crossing the boundary from right to left a reversal of the stacking sequence occurred resulting in a portion of the region behind the boundary having a twin orientation. This was likely a straininduced boundary migration process driven by the localized dislocation density gradient across the interface, which is shown in Figure 1c. The difference in dislocation densities along this line equate to a stored energy gradient of $4.22 \mathrm{MJ} / \mathrm{m}^{3}$.

In case 2 (Figure 2), a relatively dislocation-free twin has nucleated from an interface with a strain gradient at the edge of the recrystallization front and propagated into the adjacent unrecrystallized region, consuming the stored energy in its path. The stored energy gradient between the twin and the region into which it is growing along the line shown in Figure $2 b$ is $3.66 \mathrm{MJ} / \mathrm{m}^{3}$. This is indicative of some variation of the popping out model, where a segment of random high angle boundary dissociates into twin and twin related boundaries through the emission of partial dislocations. Meyers and Murr's original theory suggests that a decrease in total interfacial energy is the driving force for this mechanism [9], however, the current results indicate that stored energy gradients, such as those at the edge of a recrystallization front [27,29], may also play a role. This is in good agreement with the observation of Field, et al. of twins forming out from recrystallization fronts into strained regions when there is no longer enough driving force for the interface itself to migrate [14].

In the third case (Figure 3) defect-free twins were observed in the interior of high dislocation density grain, far from any visible boundaries, although they may have nucleated at a boundary above or below the cross section of the microstructure preserved in the TEM specimen. Two relatively dislocation-free twin domains can be seen in the interior of a deformed grain, with the highest concentration of dislocations directly between the twins. This is indicative of deformation twinning because existing annealing twin formation models require nucleation at an existing grain boundary, whereas deformation twinning does not. Additionally, the location of the peak dislocation density between the twins indicates that this region was subject to a high local shear stress that resulted in more plasticity than occurred in the surrounding region.

Three distinct dislocation density gradient configurations were observed at the sites of twin nucleation in $\mathrm{Cu}$. The twin structures produced are equivalent but the local stored energy gradients are indicative of different nucleation mechanisms. The presence of all three cases in similar microstructures demonstrates that more than one twin formation mechanism may be activated during typical thermomechanical processes based on the local strain distributions present in a given region of the microstructure. Therefore, these results suggest that no single model of annealing twin formation, such as the probabilistic growth accident model, can accurately predict twin densities on a global scale as long as local deviations in strain or dislocation density give rise to multiple, unique twin nucleation events within a given microstructure. PED-TEM coupled with Nye tensor analysis provides a quantitative approach to 
understanding twin nucleation that can be used to identify the driving forces for twin nucleation mechanisms.

Figure 1. a) TEM micrograph of twin nucleated at grain boundary. b) [100] inverse pole figure map corresponding to the region shown in a. c) Dislocation density map corresponding to the region shown in a. d) Dislocation density profile along the arrow shown in b. Dashed line indicates the location of the grain boundary.

Figure 2. a) TEM micrograph of twin (indicated by red arrow) nucleated at grain boundary. b) [100] inverse pole figure map corresponding to the region shown in a. c) Dislocation density map corresponding to the region shown in a. cd) Dislocation density profile along the arrow shown in b. Dashed lines indicate the location of the twin boundaries.

Figure 3. a) TEM micrograph of two high aspect ratio twins in a grain interior. b) [100] inverse pole figure map corresponding to the region shown in a. c) Dislocation density map corresponding to the region shown in a. d) Dislocation density profile along the arrow shown in b. Dashed lines indicate the location of the twin boundaries.

\section{Acknowledgements}

The authors gratefully acknowledge funding from the United States Department of Energy, Basic Energy Sciences, under the Early Career program (contract DE-SC0008274).

The authors would also like to acknowledge Christopher R. Weinberger for our useful discussions.

\section{References}

[1] V. Randle, Acta Mater. 52 (2004) 4067-4081.

[2] C. Hu, S. Xia, H. Li, T. Liu, B. Zhou, W. Chen, N. Wang, Corros. Sci. 53 (2011) 1880 1886. 
[3] C.M. Barr, G.A. Vetterick, K.A. Unocic, K. Hattar, X.-M. Bai, M.L. Taheri, Acta Mater. 67 (2014) 145-155.

[4] C.M. Barr, L. Barnard, J.E. Nathaniel, K. Hattar, K.A. Unocic, I. Szlurfarska, D. Morgan, M.L. Taheri, J. Mater. Res. 30 (2015) 1-10.

[5] M. Kumar, A.J. Schwartz, W.E. King, Acta Mater. 50 (2002) 2599-2612.

[6] Y. Chong, Z. Liu, A. Godfrey, W. Liu, Y. Weng, Metall. Mater. Trans. E 1 (2014) 58-66.

[7] M. Shimada, H. Kokawa, Z.J. Wang, Y.S. Sato, I. Karibe, Acta Mater. 50 (2002) 23312341.

[8] H. Gleiter, Acta Metall. 17 (1969) 1421-1428.

[9] M. Meyers, L. Murr, Acta Metall. 26 (1978) 951-962.

[10] S. Mahajan, C.S. Pande, M.A. Imam, B.B. Rath, Acta Mater. 45 (1997) 2633-2638.

[11] J.R. Cahoon, Q. Li, N.L. Richards, Mater. Sci. Eng. A 526 (2009) 56-61.

[12] J. Christian, S. Mahajan, Prog. Mater. Sci. 39 (1995) 1-157.

[13] I.J. Beyerlein, X. Zhang, A. Misra, Annu. Rev. Mater. Res. 44 (2014) 329-363.

[14] D.P. Field, L. Bradford, M. Nowell, T. Lillo, Acta Mater. 55 (2007) 4233-4241.

[15] W. Wang, S. Lartigue-Korinek, F. Brisset, a. L. Helbert, J. Bourgon, T. Baudin, J. Mater. Sci. 50 (2014) 2167-2177.

[16] Y. Jin, B. Lin, M. Bernacki, G.S. Rohrer, A.D. Rollett, N. Bozzolo, Mater. Sci. Eng. A 597 (2014) 295-303.

[17] J.G. Brons, G.B. Thompson, Acta Mater. 61 (2013) 3936-3944.

[18] J.L. Bair, S.L. Hatch, D.P. Field, Scr. Mater. 81 (2014) 52-55.

[19] V. Randle, Acta Mater. 47 (1999) 4187-4196.

[20] X. Fang, K. Zhang, H. Guo, W. Wang, B. Zhou, Mater. Sci. Eng. A 487 (2008) 7-13.

[21] X. Fang, W. Wang, Z. Cai, C. Qin, B. Zhou, Mater. Sci. Eng. A 527 (2010) 1571-1576.

[22] C.M. Barr, E. Anber, J. Hsieh, M.L. Taheri, Corros. Sci. In Review (2016).

[23] A.C. Leff, C.R. Weinberger, M.L. Taheri, Ultramicroscopy 153 (2015) 9-21.

[24] J. Jiang, T. Zhang, F.P.E. Dunne, T. Ben Britton, Proc. R. Soc. A Math. Phys. Eng. Sci. 472 (2016) 20150690.

[25] a Arsenlis, D.. Parks, Acta Mater. 47 (1999) 1597-1611.

[26] J. Jiang, T.B. Britton, A.J. Wilkinson, Ultramicroscopy 125 (2012) 1-9.

[27] M. Taheri, H. Weiland, A. Rollett, Metall. Mater. Trans. A 37 (2006) 19-25.

[28] E.F. Rauch, M. Véron, Mater. Charact. 98 (2014) 1-9.

[29] M.L. Taheri, D. Molodov, G. Gottstein, a. D. Rollett, Zeitschrift Für Met. 96 (2005) $1166-1170$. 
A)

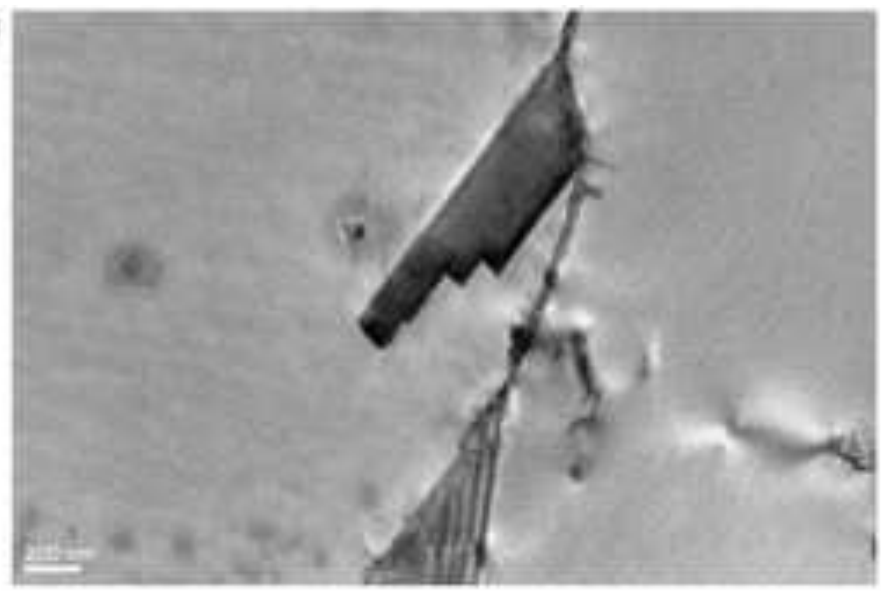

B)

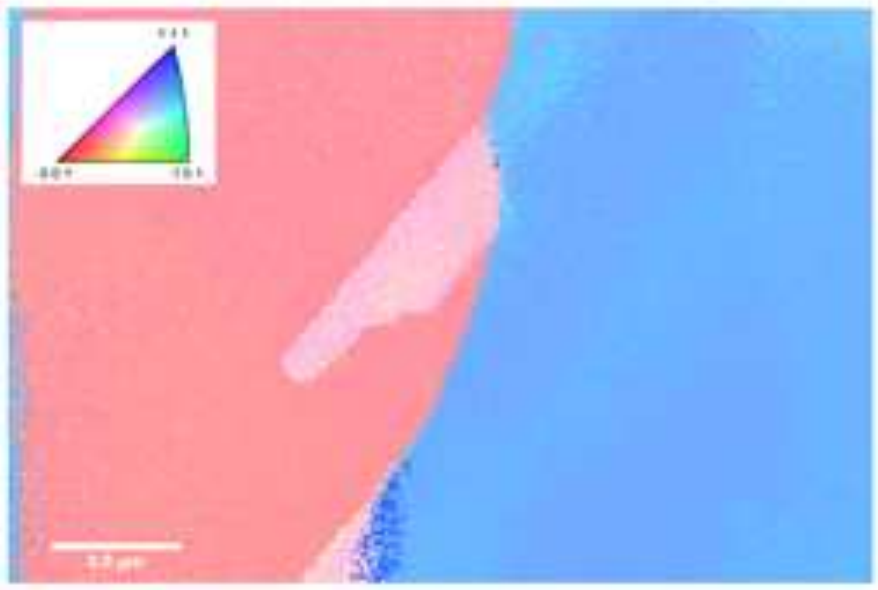

C)
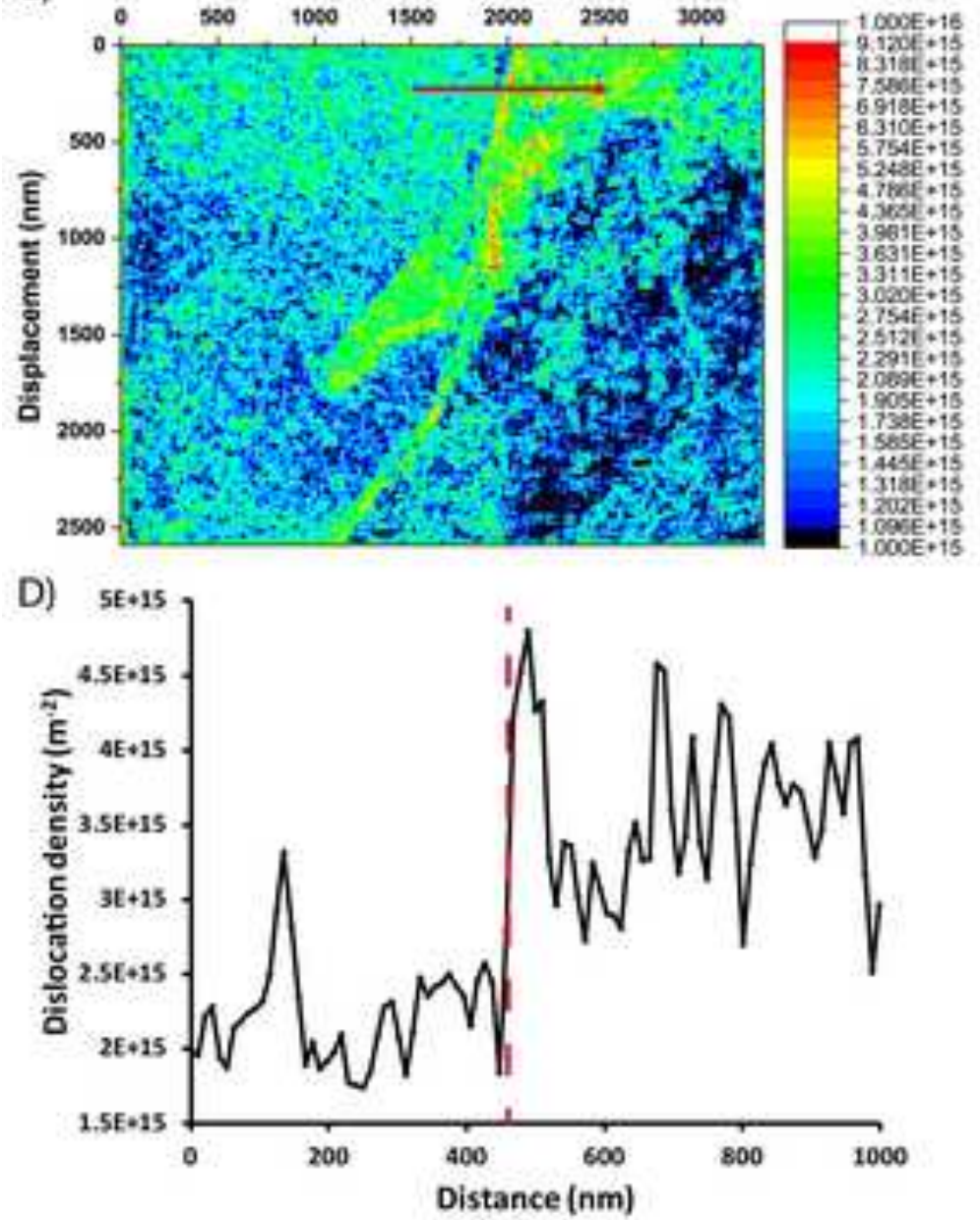
A)

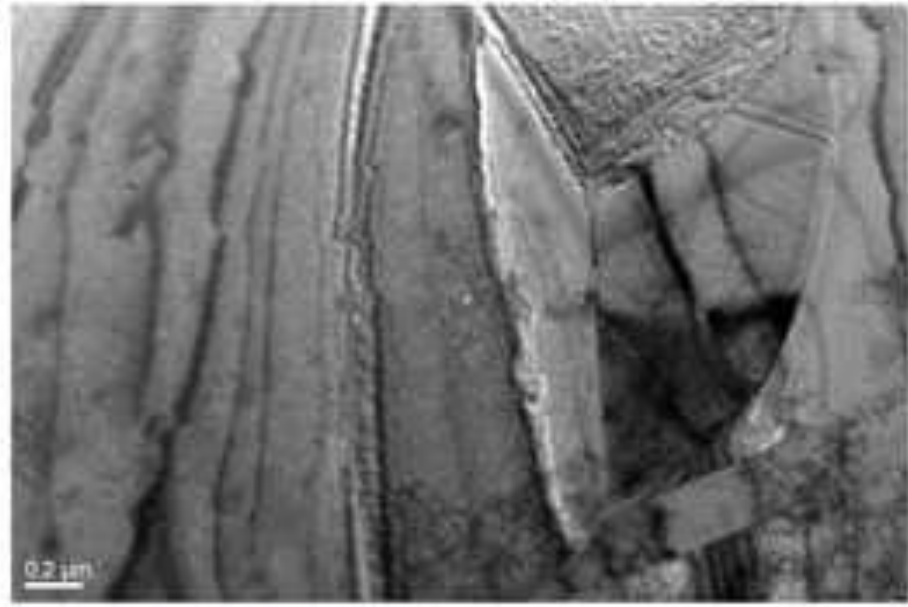

B)
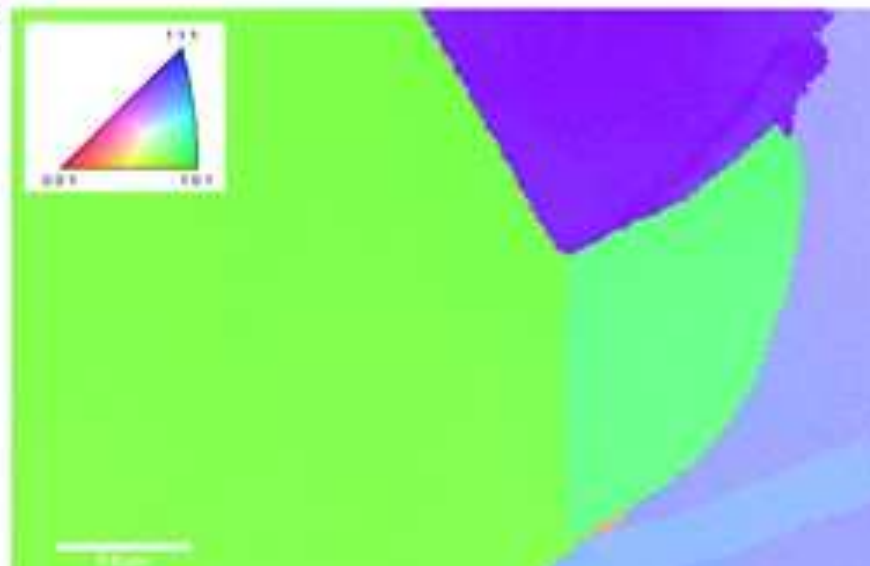

C)
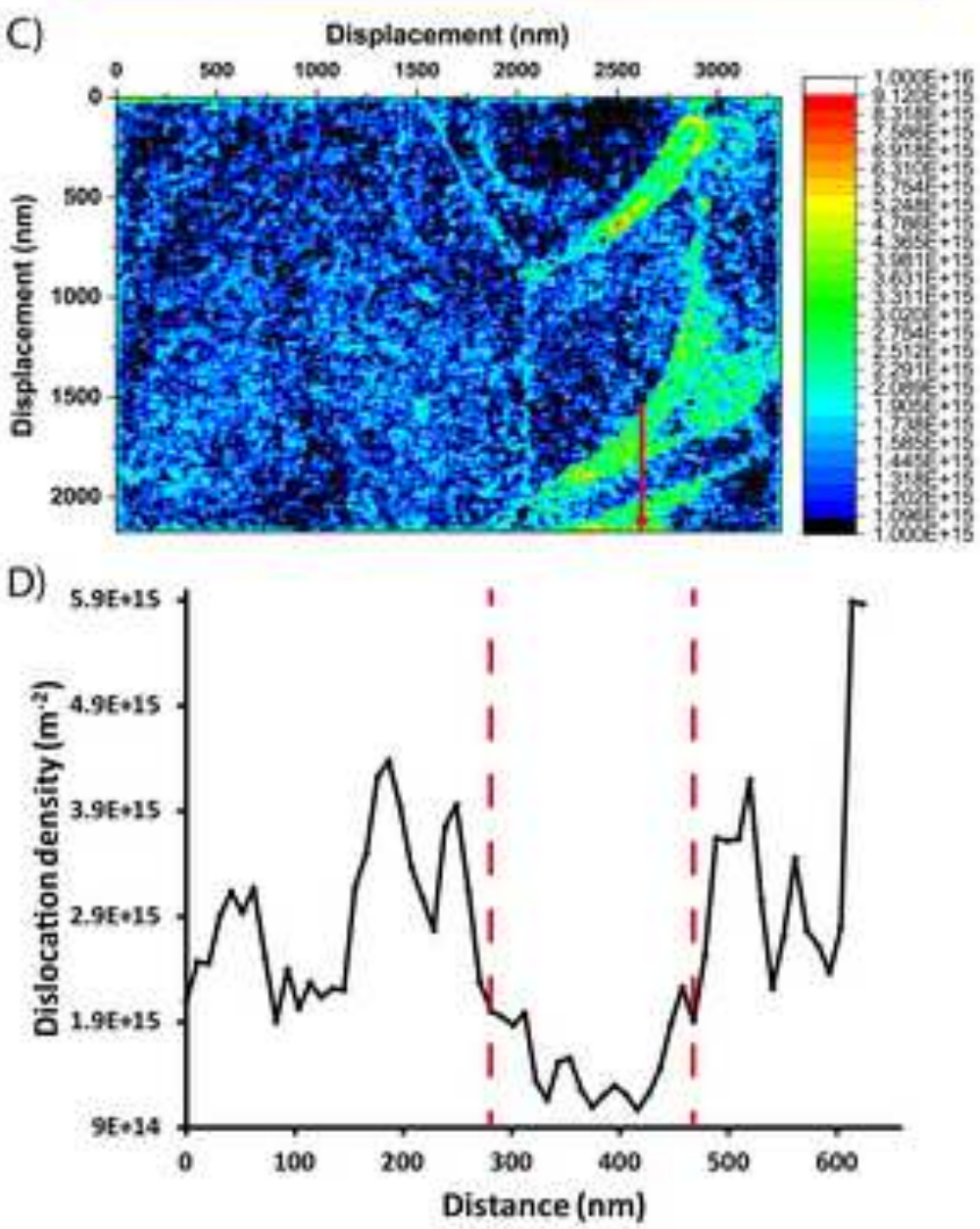
A)

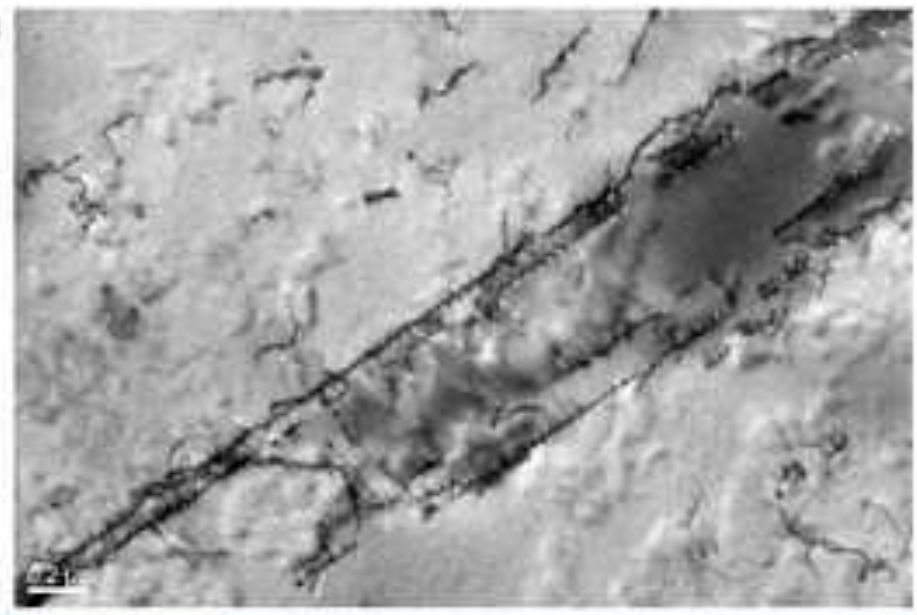

B)
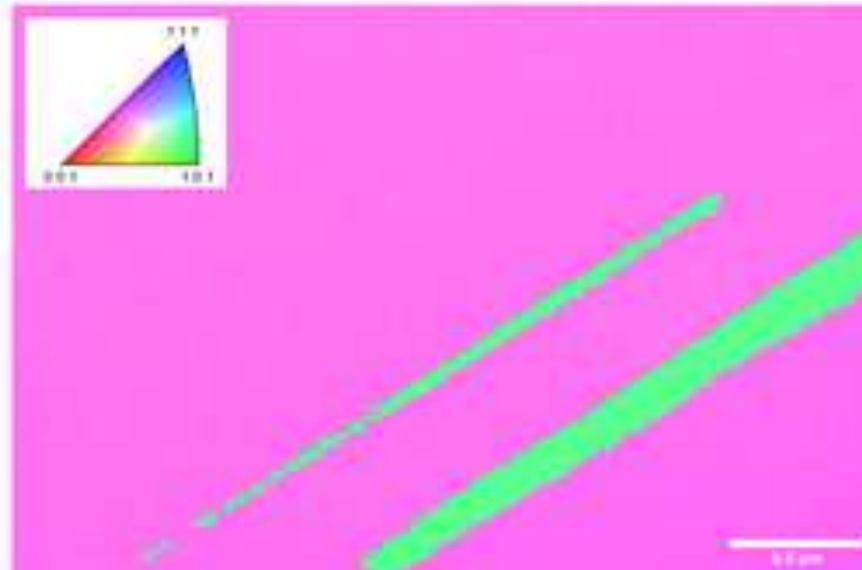

C)
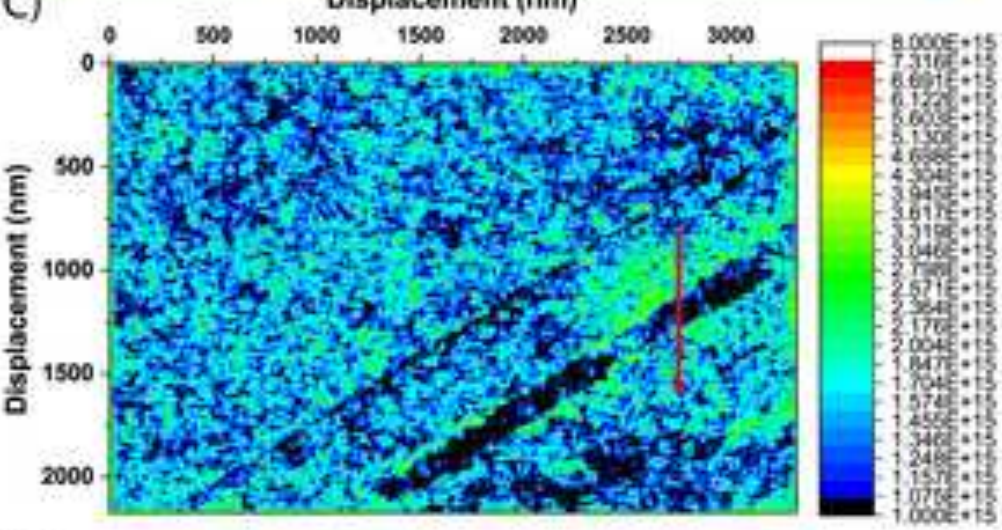

D)

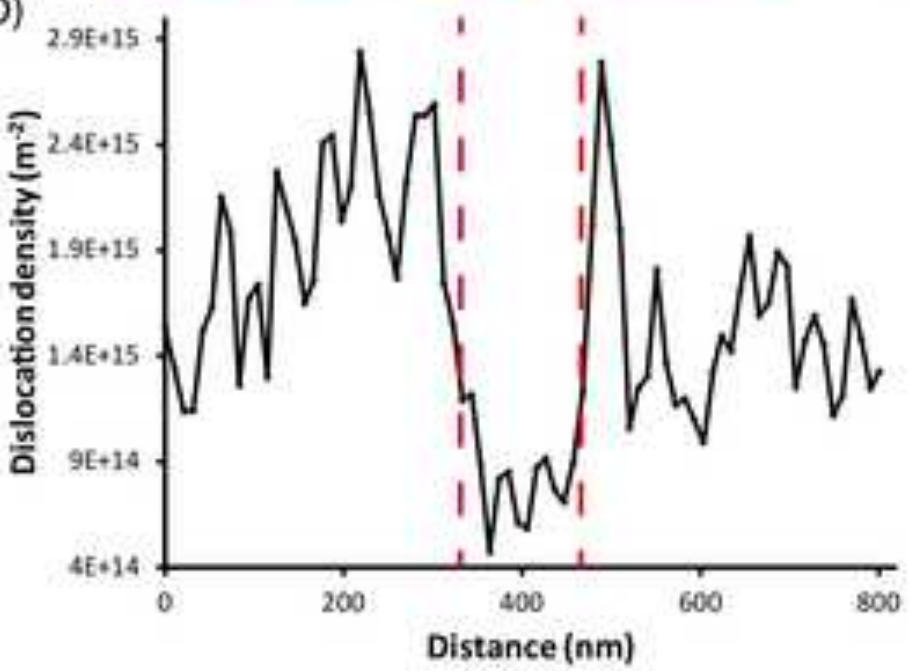

